\title{
Lower bounds for periods of Ducci sequences
}

\author{
Florian Breuer \\ School of Mathematical and Physical Sciences, University of Newcastle, Newcastle, NSW 2308, Australia \\ florian.breuer@newcastle.edu.au \\ Igor E. Shparlinski \\ School of Mathematics and Statistics, University of New South Wales, Sydney, NSW 2052, Australia \\ igor.shparlinski@unsw.edu. au
}

\begin{abstract}
A Ducci sequence is a sequence of integer $n$-tuples obtained by iterating the map

$$
D:\left(a_{1}, a_{2}, \ldots, a_{n}\right) \mapsto\left(\left|a_{1}-a_{2}\right|,\left|a_{2}-a_{3}\right|, \ldots,\left|a_{n}-a_{1}\right|\right) .
$$

Such a sequence is eventually periodic and we denote by $P(n)$ the maximal period of such sequences for given $n$. We prove lower bounds for $P(n)$ by counting certain partitions.
\end{abstract}

\section{Introduction}

Let $n$ be a positive integer. A Ducci sequence is a sequence of integer $n$-tuples obtained by iterating the map

$$
D: \mathbb{Z}^{n} \rightarrow \mathbb{Z}^{n}
$$

defined as follows:

$$
D:\left(a_{1}, a_{2}, \ldots, a_{n}\right) \mapsto\left(\left|a_{1}-a_{2}\right|,\left|a_{2}-a_{3}\right|, \ldots,\left|a_{n}-a_{1}\right|\right) .
$$

There is a long literature on Ducci sequences, see for example [BLM07, BM08, Bre19, CM37, CST05, Cla18, Ehr90, Lud81, MST06, SB18] and the references therein.

Ducci sequences are eventually periodic, and for each $n$ the largest period is denoted by $P(n)$; it is the period of the sequence starting with $(0,0, \ldots, 0,1)$. The sequence $P(1), P(2), \ldots$ is entry A038553 in the Online Encyclopedia of Integer Sequences [OEIS]. Since $P\left(2^{k}\right)=1$ and $P\left(2^{k} m\right)=2^{k} P(m)$ if $m$ is not a power of 2 , by [Ehr90, Theorem 4], we restrict our attention to odd $n$.

The following upper bounds on $P(n)$ are known. Denote by $t=\operatorname{ord}_{(\mathbb{Z} / n \mathbb{Z})^{*}}(2)$ the multiplicative order of 2 modulo $n$. If there exists an integer $M$ for which $2^{M} \equiv-1 \bmod n$, then we say $n$ is 'with -1 '. The first of the following upper bounds is proved in [Lud81], the second in [Ehr90] and the third in [Bre19]. 
It is convenient to introduce the following quantities

$$
B_{1}(n)=2^{t}-1 \quad \text { and } \quad B_{2}(n)=n\left(2^{t / 2}-1\right)
$$

Theorem A Let $n$ be an odd integer, and the multiplicative order of 2 modulo $n$. Then,

1. $P(n)$ divides $B_{1}(n)$.

2. Suppose $n$ is with -1 , then $P(n)$ divides $B_{2}(n)$.

3. Suppose that $n=p^{k}$ with $p \equiv 5 \bmod 8$ prime and 2 is a primitive root modulo $p^{k}$. If the equation $x^{2}-p y^{2}=-4$ has no solutions in odd integers $x, y \in \mathbb{Z}$, then $P(n)$ divides $\frac{1}{3} B_{2}(n)$.

As for lower bounds, the first of the following results is found again in [Ehr90], and the remaining ones in [GS95].

Theorem B Let $n$ be an odd integer. Then

1. $n$ divides $P(n)$.

2. $P(n)=n$ if and only if $n=2^{r}-1$ for some positive integer $r$.

3. If $n$ is with -1 , then $P(n) \geqslant n(n-2)$.

4. If $n$ is with -1 , then $P(n)=n(n-2)$ if and only if $n=2^{r}+1$ for some positive integer $r$.

The goal of the present paper is to prove new asymptotic lower bounds for $P(n)$ in terms of $t$ and $n$. Our starting point is the fact from [BLM07] that $P(n)$ is the lowest common multiple of multiplicative orders of elements $\zeta+1$, where $\zeta$ is a primitive $n$th root of unity in the finite field $\mathbb{F}_{2^{t}}$.

Since our results require that at least $t>\sqrt{2 n}$ holds, in Section 4 we also give a short survey of known results about the size of $t$.

\section{Multiplicative orders and partitions}

Let $1 \leqslant a<n$ be an integer prime to $n$. 
Consider the set of representatives, chosen in the interval $[1, n]$, of the coset $a\langle 2\rangle \subseteq$ $(\mathbb{Z} / n \mathbb{Z})^{*}$ of the multiplicative group $\langle 2\rangle$ generated by 2 in the residues ring modulo $n$. That is,

$$
\begin{aligned}
\mathcal{S}_{a, n}:=\left\{j \in \mathbb{Z}_{>0}: 1 \leqslant j \leqslant n,\right. & \operatorname{gcd}(j, n)=1, \\
& \left.\exists e_{j} \in \mathbb{Z}_{\geqslant 0}, j \equiv a 2^{e_{j}} \bmod n\right\}
\end{aligned}
$$

Its cardinality is $\# \mathcal{S}_{a, n}=t$.

Next, we consider the set of partitions of numbers $\leqslant t-1$ into distinct parts from $\mathcal{S}_{a, n}:$

$$
\mathcal{P}_{a, n}:=\left\{\left(u_{j}\right)_{j \in \mathcal{S}_{a, n}} \in\{0,1\}^{t} \mid \sum_{j \in \mathcal{S}_{a, n}} u_{j} j \leqslant t-1\right\}
$$

Our main result is

Theorem 2.1 Suppose $n$ is odd and a is relatively prime to $n$. Then $P(n) \geqslant \# \mathcal{P}_{a, n}$.

Proof. It follows from [BLM07, Theorem 3.9] that $P(n)$ is the lowest common multiple of the multiplicative orders of $\zeta+1$, where $\zeta$ ranges over all $n$th roots of unity $1 \neq \zeta \in \mathbb{F}_{2^{t}}$.

Let $\zeta \in \mathbb{F}_{2^{t}}$ be a primitive $n$th root of unity. The idea is to show that every partition in $\mathcal{P}_{a, n}$ leads to a distinct power of $\zeta+1$. For this we follow the strategy of [ASV10].

Let $u=\left(u_{j}\right)_{j \in \mathcal{S}_{a, n}} \in \mathcal{P}_{a, n}$, and set

$$
Q_{u}=\sum_{j \in \mathcal{S}_{a, n}} u_{j} 2^{e_{j}}
$$

where $j \equiv a 2^{e_{j}} \bmod n$. We also choose an integer $b$ for which $a b \equiv 1 \bmod n$. Now

$$
\begin{aligned}
(\zeta+1)^{Q_{u}} & =\prod_{j \in \mathcal{S}_{a, n}}(\zeta+1)^{u_{j} 2^{e_{j}}}=\prod_{j \in \mathcal{S}_{a, n}}\left(\zeta^{2^{e_{j}}}+1\right)^{u_{j}} \\
& =\prod_{j \in \mathcal{S}_{a, n}}\left(\zeta^{b j}+1\right)^{u_{j}}=\prod_{j \in \mathcal{S}_{a, n}}\left(\vartheta^{j}+1\right)^{u_{j}},
\end{aligned}
$$

where $\vartheta=\zeta^{b} \in \mathbb{F}_{2^{t}}$ is another primitive $n$th root of unity.

Let

$$
v=\left(v_{j}\right)_{j \in \mathcal{S}_{a, n}} \in \mathcal{P}_{a, n}
$$

be another partition distinct from $u$, we must show that $v$ gives rise to a distinct power of $\zeta+1$. Suppose $(\zeta+1)^{Q_{u}}=(\zeta+1)^{Q_{v}}$, so

$$
\prod_{j \in \mathcal{S}_{a, n}}\left(\vartheta^{j}+1\right)^{u_{j}}=\prod_{j \in \mathcal{S}_{a, n}}\left(\vartheta^{j}+1\right)^{v_{j}} .
$$


Denote by $f(X) \in \mathbb{F}_{2}[X]$ the minimal polynomial of $\vartheta$; it has degree $t$. Then $f(X)$ must divide $U(X)-V(X)$, where

$$
U(X)=\prod_{j \in \mathcal{S}_{a, n}}\left(X^{j}+1\right)^{u_{j}} \quad \text { and } \quad V(X)=\prod_{j \in \mathcal{S}_{a, n}}\left(X^{j}+1\right)^{v_{j}} .
$$

Since these polynomials have degree $\leqslant t-1<\operatorname{deg} f$ it follows that $U(X)=V(X)$. After removing common factors from both polynomials (corresponding to $u_{j}=v_{j}$ ), we obtain the identity

$$
\prod_{h \in \mathcal{H}}\left(X^{h}+1\right)^{u_{h}}=\prod_{k \in \mathcal{K}}\left(X^{k}+1\right)^{v_{k}}
$$

where $\mathcal{H}$ and $\mathcal{K}$ are disjoint subsets of $\mathcal{S}_{a, n}$. But now we find that the term of smallest positive degree is $x^{e}$ where $e$ is the smallest element of $\mathcal{H} \cup \mathcal{K}$, but this only appears on one side of the identity (2.2). This contradiction concludes the proof.

Remark 2.2 Some parts of the proof of Theorem 2.1 can be shortened by appealing to [Pop14, Lemma 1], however for completeness and since [Pop14] may not be easily accessible, we present a full self-contained proof.

\section{Counting partitions}

Now we construct lower bounds for the cardinality of $\mathcal{P}_{a, n}$ for $n$ of prescribed arithmetic structure. As we have mentioned, these bounds are only useful if $t$ is not too small, specifically $t>\sqrt{2 n}$.

Suppose first that $t=\varphi(n)$, that is, 2 is a primitive root modulo $n$. In this case, $n=p^{k}$ must be a power of an odd prime $p$.

When $n=p$, we find that $\mathcal{P}_{a, n}$ contains the set of partitions of $n-2$ into distinct parts, and the standard asymptotic for that gives (see e.g. [And76, Theorem 6.4])

Corollary 3.1 Suppose $n=p$ is an odd prime and 2 is a primitive root modulo $p$. Then, as $n \rightarrow \infty$,

$$
P(n) \geqslant \exp \left[\left(\frac{\pi}{\sqrt{3}}+o(1)\right) \sqrt{n}\right]
$$

The case of Corollary 3.1 is already contained in [Pop12, Theorem 1]; in particular, the completely explicit lower bound (for 2 a primitive root modulo $n=p$ )

$$
P(n) \geqslant(80(n-2))^{-\sqrt{2}} \exp \left(\pi \sqrt{\frac{n-2}{3}}\right)
$$


follows from [Pop12, Corollary 4], see also [Pop14] for some related results.

Next, suppose that $n=p^{k}$ and 2 is a primitive root modulo $n$. For this it suffices that 2 is a primitive root modulo $p$ and $p$ is not a Wieferich prime, that is, $2^{p-1} \not \equiv 1 \bmod p^{2}$.

We have $t=p^{k-1}(p-1)$ and $\mathcal{P}_{a, n}$ contains the set of partitions of $t-1$ into distinct parts which are not divisible by $p$. An asymptotic formula for the number of such partitions appears in [Hag64, Corollary 7.2], and we obtain

Corollary 3.2 Fix an odd non-Wieferich prime $p$ and suppose that 2 is a primitive root modulo $p$. Let $n=p^{k}$, then as $k \rightarrow \infty$, we have

$$
P(n) \geqslant \exp \left[\left(\frac{\pi}{\sqrt{3}} \sqrt{\frac{p-1}{p}}+o(1)\right) \sqrt{n}\right] .
$$

If $t<\varphi(n)$, then, inspired by [GS98], we estimate the cardinality of $\mathcal{P}_{a, n}$ as follows. Let $2 \leqslant N<t$ be an integer, and denote by $\mathcal{S}_{a, n}(N)=\mathcal{S}_{a, n} \cap[1, N]$. Each subset $\mathcal{J} \subseteq \mathcal{S}_{a, n}(N)$ of cardinality $\# \mathcal{J}=J \leqslant t / N$ produces a valid partition $u \in \mathcal{P}_{a, n}$, where $u_{j}=1$ if $j \in \mathcal{J}$ and $u_{j}=0$ otherwise. Thus we obtain

$$
\# \mathcal{P}_{a, n} \geqslant \sum_{J \leqslant t / N}\left(\begin{array}{c}
\# \mathcal{S}_{a, n}(N) \\
J
\end{array}\right)
$$

It remains to estimate $\# \mathcal{S}_{a, n}(N)$ and choose suitable $a$ and $N$.

It is well known that,

$$
\#\{j: 1 \leqslant j \leqslant N, \operatorname{gcd}(j, n)=1\}=N \varphi(n) / n+O\left(n^{o(1)}\right)
$$

see, for example, [Shp18, Lemma 2.1].

Now among the cosets of $\langle 2\rangle \subseteq(\mathbb{Z} / n \mathbb{Z})^{*}$, at least one must have at least the average number of representatives in $[1, N]$, so there exists an integer $a$, prime to $n$, for which

$$
\begin{aligned}
\# \mathcal{S}_{a, n}(N) & \geqslant \frac{t}{\varphi(n)} \cdot \#\{j: 1 \leqslant j \leqslant N, \operatorname{gcd}(j, n)=1\} \\
& =\frac{t}{\varphi(n)}\left(N \varphi(n) / n+O\left(n^{o(1)}\right)\right)=(1+o(1)) \frac{t N}{n}
\end{aligned}
$$

as $n \rightarrow \infty$, provided $N \geqslant n^{\varepsilon}$ for some fixed $\varepsilon>0$.

Now we choose $N=\lfloor\sqrt{2 n}\rfloor$. Since $t \geqslant n^{1 / 2+\varepsilon}$, we have

$$
\# \mathcal{S}_{a, n}(N) \geqslant \frac{t N}{n}+O\left(n^{o(1)}\right)=(2+o(1)) \frac{t}{N} .
$$


Thus by the Stirling formula

$$
\begin{aligned}
\# \mathcal{P}_{a, n} & \geqslant \sum_{J \leqslant t / N}\left(\begin{array}{c}
\# \mathcal{S}_{a, n}(N) \\
J
\end{array}\right) \geqslant\left(\begin{array}{c}
\# \mathcal{S}_{a, n}(N) \\
\lfloor t / N\rfloor
\end{array}\right) \\
& \geqslant \exp \left((2 \log 2+o(1)) \frac{t}{N}\right) .
\end{aligned}
$$

Thus we have proved

Corollary 3.3 Suppose $n$ is odd and $t$ is the multiplicative order of 2 modulo $n$. Then

$$
P(n) \geqslant \exp \left[(\log 4+o(1)) \frac{t}{\sqrt{2 n}}\right] .
$$

In particular, if $n=p^{k}$ then it is easy to show that $t \geqslant c(p) p^{k}$, where $c(p)>0$ depends only on $p$, hence Corollary 3.3 gives a version of Corollary 3.2 in the form

$$
P(n) \geqslant \exp (c(p) \sqrt{n}) .
$$

We remark that the condition $t>\sqrt{2 n}$ of Corollary 3.3 corresponds to the limits of our method. Indeed, there are about $\varphi(n) / t$ distinct cosets $\mathcal{S}_{a, n}$ and since $\varphi(n)=n^{1+o(1)}$ each of them is expected to contain very few elements from the interval $[1, t]$ which are the only suitable elements which can be used in the construction of the set $\mathcal{P}_{a, n}$ given by $(2.1)$.

Since

$$
\frac{\log 4}{\sqrt{2}} \approx 0.98025 \quad \text { and } \quad \frac{\pi}{\sqrt{3}} \approx 1.8138,
$$

in the case of $t \approx n$ we recover a result similar to Corollaries 3.1 and 3.2, but with a smaller constant in the exponent.

Our lower bounds are quite small compared to the upper bounds $P(n) \leqslant B_{1}(n) \sim 2^{t}$ and $P(n) \leqslant B_{2}(n) \sim n 2^{t / 2}$, see (1.1), which follow from Theorem A. On the other hand, they are typically much stronger than linear and quadratic in $n$ lower bounds which one can extract from Theorem B.

\section{Lower bounds on multiplicative orders}

Since the quality of our bounds depends rather dramatically on the multiplicative order of 2 modulo $n$, here we give a short outline of known results. 
First we observe that the applicability of Corollary 3.1 for infinitely many prime $n=p$ is equivalent to Artin's conjecture, see [Mor12] for an exhaustive survey. On the other hand, we are not aware of any conditional (and certainly unconditional) results or wellestablished conjectures towards a version of Artin's conjecture for non-Wieferich primes which appear Corollary 3.2. It is natural to expect that there are infinitely many such primes but known results are scarce [Sil88].

Primes $p$ and integers $n$ for which $t$ is large, in particular exceeds $\sqrt{p}$, have been studied in many different contexts, but most commonly in the theory of pseudorandom number generators. These results originate from the work of Erdös and Murty [EM99] and are conveniently summarised in [KP05]. For example, for any function $\psi(n) \rightarrow 0$ as $n \rightarrow \infty$ we have $t \geqslant n^{1 / 2+\psi(n)}$ for almost all (in a sense of relative density) primes $p=n$ (see [EM99, Theorem 1]) and odd integers $n$ (see [KP05, Theorem 11]). Furthermore, for a positive proportion of primes $p=n$ (see [KP05, Lemma 19])) and odd integers $n$ (see [KP05, Theorem 21]) we have $t \geqslant n^{0.677}$.

\section{Numerical results}

It is interesting to compare the lower bound of Theorem 2.1 with actual values of $P(n)$. Table 5.1 shows numerical values of $P(n)$ and $\# \mathcal{P}_{a, n}$ for odd $n \leqslant 101$ and a representative $a$ for each coset $(\mathbb{Z} / n \mathbb{Z})^{*} /\langle 2\rangle$. Unsurprisingly, the largest value of $\# \mathcal{P}_{a, n}$ is achieved for $a=1$ in these small cases, due to the presence of small powers of two in $\mathcal{S}_{a, n}$. However, when $n=109$, we find that

$$
\# \mathcal{P}_{1,109}=99<178=\# \mathcal{P}_{3,109}=\max _{\operatorname{gcd}(a, 109)=1} \# \mathcal{P}_{a, 109} .
$$

These values were computed using Sage. 

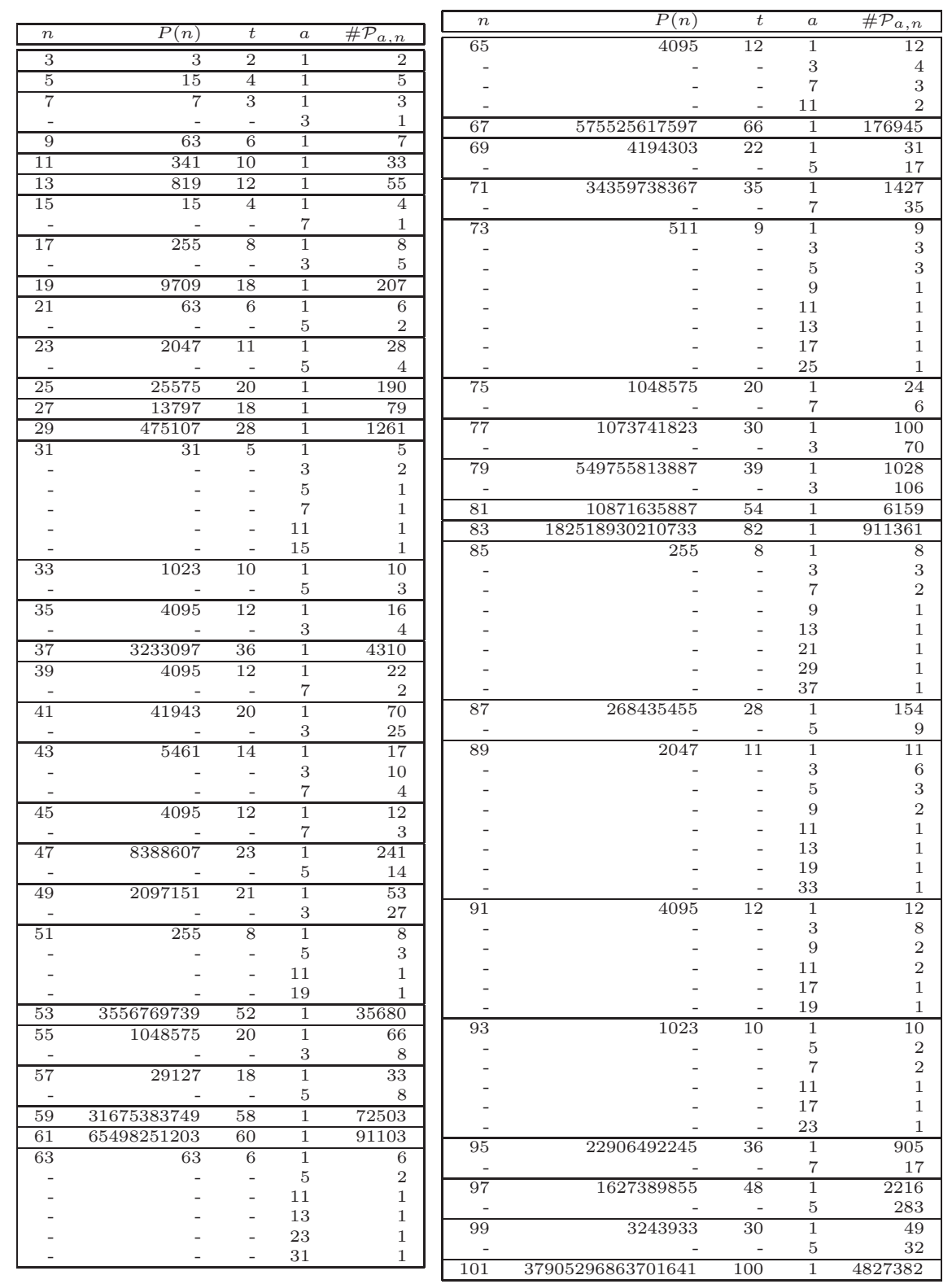

Table 5.1: Values of $P(n)$ and $\# \mathcal{P}_{a, n}$ for odd $n \leqslant 101$. 
Acknowledgments The first author thanks the Alexander-von-Humboldt Foundation for support, the Universität Heidelberg for hospitality and Hannes Breuer for interesting discussions. The second author was supported in part by the Australian Research Council Grant DP180100201.

The authors are grateful to the organisers of the the Sixth Number Theory Down Under Conference (NTDU-6), Canberra, 24-27 September, 2018, for creating a very encouraging and collaborative atmosphere, which has led to this work.

\section{References}

[ASV10] O. Ahmadi, I. E. Shparlinski and J. F. Voloch, Multiplicative order of Gauss periods, Internat. J. Number Theory 6 (2010) 877-882. 3

[And76] G. E. Andrews, The Theory of Partitions, Addison-Wesley, New York, 1976. 4

[BLM07] F. Breuer, E. Lötter and A. B. van der Merwe, Ducci sequences and cyclotomic polynomials, Finite Fields Appl. 13 (2007), 293-304. 1, 2, 3

[Bre19] F. Breuer, Periods of Ducci sequences and odd solutions to a Pellian equation, Bull. Aust. Math. Soc. 100 (2019), 201-205. 1

[BM08] R. Brown and J. L. Merzel, The number of Ducci sequences with given period, Fibonacci Quart. 45 (2007), 115-121. 1

[CM37] C. Ciamberlini and A. Marengoni, Su una interessante curiosità numerica, Periodiche di Matematiche $\mathbf{1 7}$ (1937), 25-30. 1

[CST05] N. J. Calkin, J. G. Stevens and D. M. Thomas, A characterization for the length of cycles of the n-number Ducci game, Fibonacci Quart. 43 (2005), n 53-59. 1

[Cla18] A. Clausing, Ducci matrices, Amer. Math. Monthly 125 (2018), 901-921. 1

[Ehr90] A. Ehrlich, Periods of Ducci's N-number game of differences, Fibonacci Quart. 28 (1990), 302-305. 1, 2

[EM99] P. Erdős and M. R. Murty, On the order of $a(\bmod p)$, Proc. 5th Canadian Number Theory Association Conf., Amer. Math. Soc., Providence, RI, 1999, 87-97. 7

[GS95] H. Glaser and G. Schöffl, Ducci-sequences and Pascal's triangle, Fibonacci Quart. 33 (1995) 313-324. 2

[GS98] J. von zur Gathen and I. E. Shparlinski, Orders of Gauss periods in finite fields, Appl. Algebra Engrg. Comm. Comput. 9 (1998), 15-24. 5 
[Hag64] P. Hagis, On a class of partitions with distinct summands, Trans. Amer. Math. Soc. 112 (1964), 401-415. 5

[KP05] P. Kurlberg and C. Pomerance, On the period of the linear congruential and power generators, Acta Arith. 119 (2005), 149-169. 7

[Lud81] A. L. Ludington, Cycles of differences of integers, J. Number Theory 13 (1981), 255-261. 1

[MST06] M. Misiurewicz, J. G. Stevens and D. M. Thomas, Iterations of linear maps over finite fields, Linear Algebra Appl. 413 (2006), 218-234. 1

[Mor12] P. Moree, Artin's primitive root conjecture - A survey, Integers 12A (2012), Paper A13, 1-100. 7

[OEIS] On-line Encyclopedia of Integer Sequences, entry \#A038553. https://oeis.org/A038553. 1

[Pop12] R. Popovych, Elements of high order in finite fields of the form $\mathbb{F}_{q}[x] / \Phi_{r}(x)$, Finite Fields Appl. 18 (2012), No. 4, 700-710. 4, 5

[Pop14] R. Popovych, Sharpening of the explicit lower bounds for the order of elements in finite field extensions based on cyclotomic polynomials. Ukrainian Math. J. 66 (2014), no. 6, 916-927. 4, 5

[Shp18] I. E. Shparlinski, Linear equations with rational fractions of bounded height and stochastic matrices, Quart. J. Math. 69 ( 2018), 487-499. 5

[Sil88] J. H. Silverman, Wieferich's criterion and the abc-conjecture, J. Number Theory 30 (1988), 226-237. 7

[SB18] S. Solak and M. Bahşi, Some properties of circulant matrices with Ducci sequences, Linear Algebra Appl. 542 (2018), 557-568. 1 